\title{
A Rare Case of Ewing's Sarcoma in Pregnancy
}

\author{
Amit Kumar $^{1, \star}$, P. Sindhusha ${ }^{1}$, P. J. N. Satyavathi ${ }^{1}$, A. Vanajakshi ${ }^{1}$, S. Annapoorna ${ }^{2}$ \\ ${ }^{1}$ Department of Pharmacy Practice, Aditya College of Pharmacy, Surampalem, Andhra Pradesh, INDIA. \\ ${ }^{2}$ Department of Obstetrics and Gynecology, GSL General Hospital and Medical College, Lakshmipuram, Rajanagaram, Rajahmundry, \\ Andhra Pradesh, INDIA.
}

\begin{abstract}
Ewing's Sarcoma (ES) is a rare cancerous tumor of bone or soft tissue that usually occurs mostly in young adults (teens through mid-20s). It is very rare to diagnose ES in pregnancy. Only a few cases have been reported in a pregnant patient of ES. The treatment will be based on the size of the bone tumor. Chemotherapy is much required for the first treatment for localized ES of the bone. It is used before surgery or radiation therapy. This is called neoadjuvant chemotherapy. Here we report a rare case of a 24-year female with an Ewing's sarcoma, located in the pelvic bone detected in the $3^{\text {rd }}$ trimester of pregnancy treated successfully in G.S.L Medical College and Hospital. Overall maternal and fetal outcomes are good when treatment is administered promptly and under the care of a multi- disciplinary team.
\end{abstract}

Keywords: Chemotherapy, Ewing's sarcoma, Pregnancy, Pelvic mass, Intrauterine growth restriction.

\section{INTRODUCTION}

Ewing's Sarcoma is a part of the Ewing sarcoma family of tumor (ESFT) such as the Askin's tumor of the chest wall, Primitive neuroectodermal tumor (PNET) of bone or soft tissue, and extraskeletal Ewing sarcoma. ${ }^{1}$ It is the second most common bone tumour after osteosarcoma in children and adolescents. ${ }^{2}$ At present breast cancer is the most common tumour treated during pregnancy followed by ovarian cancer, cervical cancer, leukemia, lymphoma, and lung cancer. Bone and soft tissue sarcomas, being generally rare diseases, present more infrequently during gestation. ${ }^{3}$

\section{CASE REPORT}

A 24-year primigravida presented in 30 weeks of gestation with a two-month history of progressive, nocturnal left-side lower back and gluteal pain. The patient was treated with painkillers and antibiotics elsewhere. The pain radiated to the thigh region with painful hip movements. The patient did not have any prior medical history. On clinical examination, a mass of $8 \times 10 \mathrm{~cm}$ size was felt in the anterior and left fornix, firm in consistency, non-tender and with restricted mobility. Obstetric examination was normal. A provisional diagnosis of pelvic mass was made. Ultrasound of abdomen revealed a heterogeneous lesion of size $10.1 \times 10.9 \times 8.3$ $\mathrm{cm}$ in the pelvis extending up to paracervical region. An additional magnetic resonance imaging (MRI) of the pelvis was done which suggested a large $9.4 \times 8.6 \times 9.8 \mathrm{~cm}$ homogenous lobulated mass from the right pelvic wall, eroding the pubic rami (Figure 1). Fetal parameters were normal. All routine blood investigations were normal.

An ultrasound-guided fine-needle aspiration biopsy was performed. Histopathological examination of a biopsy of the pelvic mass showed small blue round sarcoma cells with positive immunostaining for CD99 suggestive of EWS/PNET (Figure 2).

There was no nodal involvement or spread to other organs.

At 33 weeks of gestation, the patient was referred to oncology unit for the $1^{\text {st }}$ cycle of chemotherapy. After extensive interdisciplinary consultation regarding
DOI: 10.5530/ijopp.14.4.58

Address for correspondence: Amit Kumar,

Associate professor and HOD, Department of pharmacy practice, Aditya college of pharmacy, Surampalem, Andhra pradesh, INDIA.

Phone no: +91 8790592977 Email Id: amit.britind@gmail. com

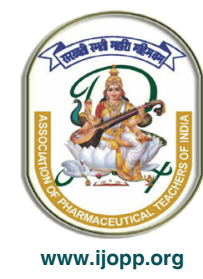




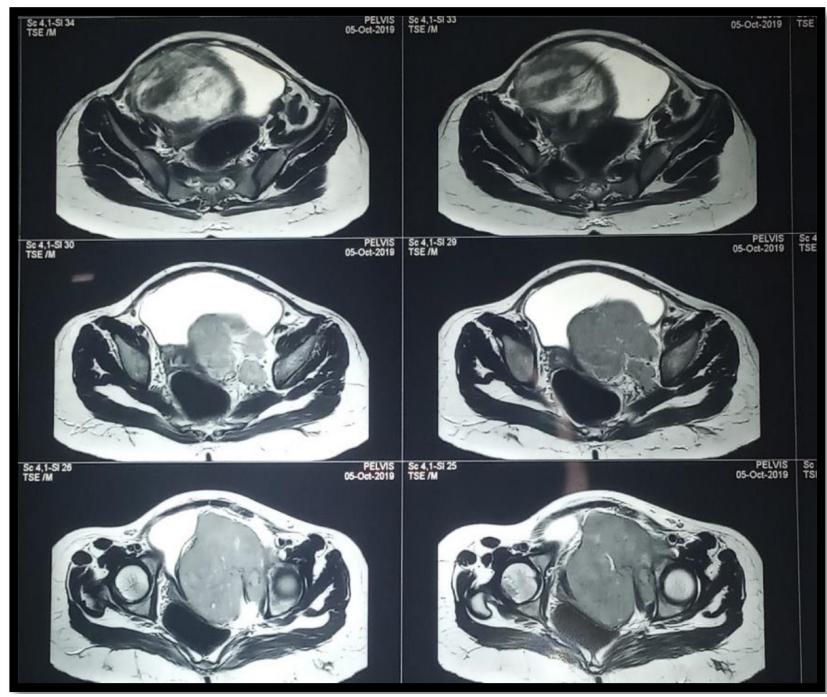

Figure 1: MRI of pelvis

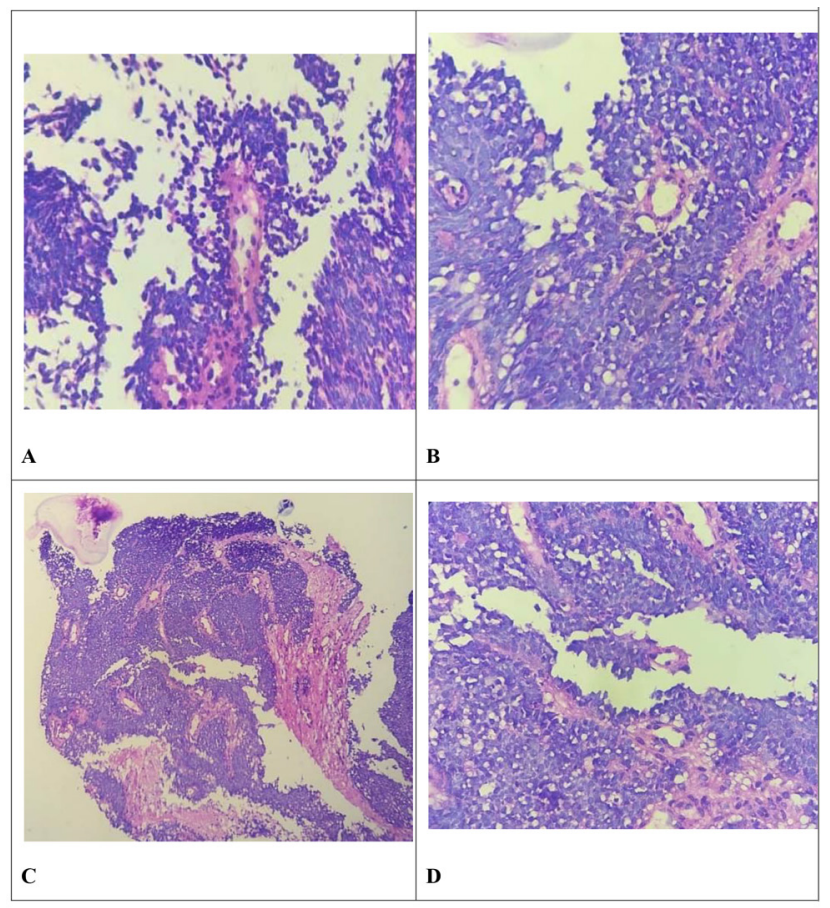

Figure 2: Fine needle aspiration biopsy of the pelvic mass.

the mother's as well as fetal prognosis, neoadjuvant chemotherapy was initiated. It consisted of VDCA Vincristine $\left(2 \mathrm{mg} / \mathrm{m}^{2} /\right.$ day), doxorubicin $\left(90 \mathrm{mg} / \mathrm{m}^{2} /\right.$ day), cyclophosphamide $\left(1400 \mathrm{mg} / \mathrm{m}^{2} /\right.$ day), actionomycin-D $\left(1.5 \mathrm{mg} / \mathrm{m}^{2} /\right.$ day) Ifosfamide $\left(2000 \mathrm{mg} / \mathrm{m}^{2} /\right.$ day $)$, and Etoposide $\left(100 \mathrm{mg} / \mathrm{m}^{2} /\right.$ day $)$ Under adequate antiemetic prophylaxis and bone marrow support, chemotherapy was well tolerated by the patient and then she was on regular follow-up. Routine antenatal workup was done. At 35 weeks 2 days of gestation, an ultrasound examination was performed which showed oligohydramnios (AFI 4-5cm) with intrauterine growth retardation. She was diagnosed to have mild anemia (hemoglobin - 9 gm \%) hence 1 unit of packed cell volume was transfused. Her hemoglobin then increased to $10.6 \mathrm{gm} \%$, total leukocyte count was 4,600 cells/cu mm. One more unit of packed cell volume was transfused. She delivered a female child of $1.87 \mathrm{~kg}$ with a 5 min Apgar score of $10 / 10$ by cesarean section. The baby was kept in the neonatal intensive care unit because of low birth weight and discharged on day 15 of birth after adequate weight gain. She was transferred back on the second day post-delivery to oncology unit for further management. There, she was given 2 cycles of chemotherapy (IE) (IE) Ifosfamide $\left(2000 \mathrm{mg} / \mathrm{m}^{2} /\right.$ day) Etoposide $\left(100 \mathrm{mg} / \mathrm{m}^{2} /\right.$ day) During chemotherapy, the patient presented with nausea and vomiting and severe anemia which was treated with erythropoiesis-stimulating agents. MRI evaluation performed after 4 cycles of chemotherapy revealed partial regression of tumor (70\% tumor shrinkage). After completion of chemotherapy, radiation was recommended for unresectable tumor (Total dose 45GY). The total treatment plan took 6 months. After 6 months follow-up, she remains disease-free and has experienced complete resolution of her pain.

\section{DISCUSSION}

Ewing's sarcoma is a highly malignant bone tumor that rapidly metastasizes at an early stage. These tumors have rapid growth and frequent distant metastasis and individuals present with chief complaint of persistent pain. Treatment options include radiation, chemotherapy, and surgery. The combination of chemotherapy with both surgery and radiotherapy increases the survival rate as compared to chemotherapy alone. Prognosis has been improved in recent years. Due to modern treatments, long-term survival up to $70 \%-80 \%$ can be reached among patients without metastases. Ewing's tumor is highly chemosensitive and hence treatment of choice is chemotherapy, but sometimes radiotherapy is also given. During pregnancy, chemotherapy is very crucial. In most of the cases, harmful effects were observed when chemotherapy was given during the $1^{\text {st }}$ trimester have an increased risk of fetal congenital malformations, intrauterine growth restriction (IUGR), or intrauterine fetal death (IUD). When chemotherapy is administered in the $2^{\text {nd }}$ trimester maternal survival rate is much better than delaying the treatment to until after delivery.

We present a case of Ewing's sarcoma which was diagnosed during pregnancy involving the pelvic bones. In this case, patient has received chemotherapy later in the course of gestation (in the $33^{\text {rd }}$ week). Most of the chemotherapy regimens were doxorubicin-based. 
The multi-agent chemotherapy protocol administered in our patient consisted of actinomycin, doxorubicin, vincristine and cyclophosphamide. The dilemma was whether to allow the patient to progress till term or to terminate immediately. Due to the advancement in chemotherapeutic agents which are better tolerated by mother and baby, we were able to achieve good neonatal outcome and optimal maternal therapy.

\section{CONCLUSION}

Ewing's sarcoma during pregnancy is extremely rare. The possibility of the treatment is decided based on a multidisciplinary approach, considering both mother and fetus avoiding excessive toxicity to the fetus with adequate treatment to the mother. The majority of published cases of Ewing's sarcoma in pregnancy use chemotherapy as the main necessity of treatment. Chemotherapy during the $3^{\text {rd }}$ trimester is relatively safe, and apart from low birth weight, there were no immediate toxic effects and no late effects within 6 months of follow-up have been reported. caesarean section is the preferred way of delivery in cases of pelvic sarcoma and possibly other tumors. In each case of pregnant women with bone or soft tissue sarcoma, a specific approach should be needed.

\section{ACKNOWLEDGEMENT}

The authors would like to thank all the staff of GSL General Hospital and Medical College, Rajahmundry for their support in the pathologic diagnosis and providing scan images.

\section{CONFLICT OF INTEREST}

The authors have no conflict of interest to declare.

\section{INFORMED CONSENT}

The patient whose case is described in the case report has provided written consent for the publication of this manuscript and any accessory images.

\section{ABBREVIATIONS}

ES: Ewing's Sarcoma; PNET: Primitive Neuroectodermal Tumour; IUGR: Intra Uterine Growth Restriction; IUD: Intra Uterine Death; MRI: Magnetic Resonance Imaging; CT: Computed Tomography.

\section{SUMMARY}

Ewing's sarcoma is a rare presentation as a pelvic mass in pregnancy and poses difficulty in diagnosis, treatment and delivery. We present such a case of Ewing's sarcoma presenting as pelvic mass in $3^{\text {rd }}$ trimester with apt diagnosis and team-based approach er were able to treat the condition simultaneously with fetal surveillance and good maternal and fetal outcome.

\section{REFERENCES}

1. Frederika fluck, L Menezes Falcao, Raquel Paxar, Isabel Pinheiro. Extra skeletal ewing sarcoma in a young patient during pregnancy. J Clin case report. 2015; 5(1).

2. Waseem khaliaq, Mohammad Farshad Bahador, Thomas Nichols Laurence, Ronald Andrew Sapiente, James Sheridan Lewis, David Lee Graham. Ewing's sarcoma: a case report of a 52-year-old woman with recurrent tumor and literature review.2012; 3(1):155-158

3. Schur S, Wild, T Brodowicz. Sarcoma of the ewing family in pregnancy: a case report of intrauterine fetal death after induction of chemotherapy. Case Rep. Oncol.2012; 5 (3): 633-638. 